\title{
BIBLIOGRAPHY
}

1. J. Dixmier, Les algèbres d'operateurs dans l'espace Hilbertien, Gauthier-Villars, Paris, 1957.

2. - Sous-anneaux abéliens maximaux dans les facteurs de type fini, Ann. of Math. 59 (1957), 279-286.

3. F. J. Murray and J. von Neumann, On rings of operators, Ann. of Math. 37 (1936), 116-229.

4. - On rings of operators. IV, Ann. of Math. 44 (1943), 716-808.

5. Sister R. J. Tauer, Maximal abelian subalgebras in finite factors of type II, Trans. Amer. Math. Soc. 114 (1965), 281-308.

The College of St. Catherine, St. Paul, Minnesota

\section{PURE SUBGROUPS HAVING PRESCRIBED SOCLES}

\section{BY PAUL HILL}

Communicated by R. S. Pierce, January 22, 1965

Let $B=\sum B_{n}$ be a direct sum of cyclic groups where, for each positive integer $n, B_{n}=\sum C\left(p^{n}\right)$ is zero or homogeneous of degree $p^{n}$ where $p$ is a fixed prime. Denote by $\bar{B}$ the torsion completion of $B$ in the $p$-adic topology. Following established terminology [1], we refer to $\bar{B}$ as the closed primary groups with basic subgroup $B$. A primary group $G$ is said to be pure-complete if each subsocle of $G$ supports a pure subgroup of $G$. A semi-complete group was defined by Kolettis in [6] to be a primary group which is the direct sum of a closed group and a direct sum of cyclic groups.

For a particular $B$, I exhibited in [3] nonisomorphic pure subgroups $H$ and $K$ of $\bar{B}$ having the same socle. Using this example, Megibben [7] was the first to show the existence of a primary group without elements of infinite height which is not pure-complete. We mention that each semi-complete group is pure-complete [4]. The purpose of this note is to announce the following theorem and corollaries; proofs will appear in another paper.

TheOREM. Suppose that $B$ is unbounded and countable and that $S$ is any proper dense subsocle of $\bar{B}$ such that $|S|=2{ }^{\star 0}$. Then $S$ supports more than $2^{\text {No }}$ pure subgroups of $\bar{B}$ which are isomorphically distinct.

The theorem has the following implications.

Corollary 1. Suppose that B is unbounded and countable and that 
$G$ is an uncountable, semi-complete, pure subgroup of $\bar{B}$ such that $B \subseteq G \subset \bar{B}$. Then there is a pure subgroup $H$ of $\bar{B}$ such that $H[p]=G[p]$ and such that $H$ is not pure-complete.

The next result shows that a conjecture stated in [8] is false.

CoRollaRy 2. The direct sum of two pure-complete groups need not be pure-complete.

A reduced primary group $G$ is said to be quasi-closed if the closure in the $p$-adic topology of each pure subgroup of $G$ is again a pure subgroup of $G$. It was shown in [5] that the class of quasi-closed groups is more general than the class of closed groups. This settled a question raised by Head in [2]. It is well known that a closed group is uniquely determined by its Ulm invariants [6]; however, we now have

CoROLlary 3. There exist more than $2^{N_{0}}$ quasi-closed groups having the same Ulm invariants.

Finally, we remark that our theorem is further evidence that the problem of classifying the pure subgroups between $B$ and $\bar{B}$ is difficult (see [1]).

\section{REFERENCES}

1. L. Fuchs, Abelian groups, Publishing House of the Hungarian Academy of Sciences, Budapest, 1958.

2. T. Head, Remarks on a problem in primary abelian groups, Bull. Soc. Math. France 91 (1963).

3. P. Hill, Certain pure subgroups of primary groups, Topics in abelian groups, Scott, Foresman and Co., Chicago, 1963.

4. P. Hill and C. Megibben, Minimal pure subgroups in primary groups, Bull. Soc. Math. France 92 (1964).

5. - Quasi-closed primary groups (to appear).

6. G. Kolettis, Semi-complete primary abelian groups, Proc. Amer. Math. Soc. 11 (1960), 200-205.

7. C. Megibben, A note on a paper of Bernard Charles, Bull. Soc. Math. France 91 (1963).

8. - Doctoral dissertation, Auburn University, Auburn, Ala., 1963.

EMORY UNIVERSITY 